\title{
Case Report Hypersensitivity Reaction and Tolerance Induction to Ethambutol
}

\author{
Josefina Rodrigues Cernadas, ${ }^{1}$ Natacha Santos, ${ }^{1}$ Claudia Pinto, ${ }^{2}$ \\ Patricia Caetano Mota, ${ }^{3}$ and Mariana Castells ${ }^{4}$
}

${ }^{1}$ Immunoallergy Department, Centro Hospitalar Sao Joao, E.P.E., 4200-319 Porto, Portugal

${ }^{2}$ Pulmonology Department, Centro Hospitalar Tras-os-Montes e Alto Douro, E.P.E., 5000-508 Vila Real, Portugal

${ }^{3}$ Pulmonology Department, Centro Hospitalar Sao Joao, E.P.E., 4200-319 Porto, Portugal

${ }^{4}$ Department of Medicine, Division of Rheumatology, Immunology, and Allergy, Brigham and Women's Hospital, Boston, USA

Correspondence should be addressed to Josefina Rodrigues Cernadas; josefinacernadas@gmail.com

Received 19 March 2013; Accepted 3 July 2013

Academic Editor: J. Mullol

Copyright (c) 2013 Josefina Rodrigues Cernadas et al. This is an open access article distributed under the Creative Commons Attribution License, which permits unrestricted use, distribution, and reproduction in any medium, provided the original work is properly cited.

\begin{abstract}
Tuberculosis remains the leading cause of death worldwide from any infectious agent and the alarming increase in the annual incidence of new cases has been described as a global emergency. Mycobacterium infection requires simultaneous administration of multiple drugs. Although the majority of treatment courses progress with minor side effects, adverse reactions to antituberculosis drugs occur in about $5 \%$ of treated patients and can be responsible for cessation or switching the therapy. Both nonimmediate (mostly maculopapular rash) and immediate reactions (urticarial reactions) have been described with these drugs. The main problem is the occurrence of reactions while the patient is on treatment with multiple drugs. The diagnosis of the culprit drug is mostly based on stopping all medication, followed by the reintroduction of each drug with a time interval of four to five days. An alternative drug should be the first approach if it is equally effective. Most of the times, none of the alternative drugs are as effective as the culprit. If this is the case, a desensitization procedure should be performed. The authors describe a case of a woman with Mycobacterium avium complex (MAC) infection, to whom treatment with ethambutol was crucial to recovery, and present a modified desensitization protocol to this drug.
\end{abstract}

\section{Case Report}

We present the case of a 66-year-old woman with a history of hypertension, dyslipidemia, chronic gastritis, depression, and non-cystic fibrosis bronchiectasis, followed in our hospital's Pulmonology Department. Her medication was losartan $100 \mathrm{mg}$ with hydrochlorothiazide $25 \mathrm{mg}$ id, omeprazole $20 \mathrm{mg}$ id, ticlopidine $250 \mathrm{mg}$ id, sertraline $50 \mathrm{mg}$ id, and inhaled fluticasone $500 \mathrm{ug}$ with salmeterol $50 \mathrm{ug}$ bid. In 2006, she was diagnosed with atypical mycobacteriosis with Mycobacterium kansasii, having completed 18 months of rifampicin, ethambutol, and isoniazid without adverse reactions. In May 2008, Mycobacterium avium complex (MAC) was identified in two sputum cultures, for which she started streptomycin (2 months), rifampicin, ethambutol, and clarithromycin. After 14 months of treatment, she presented facial erythema and angioedema of the neck and upper limbs followed by pruritic scaling maculopapular exanthema in the neck, trunk, and upper and lower limbs, associated with mild dyspnoea with no hemodynamic changes. She recurred to the hospital and was treated with intravenous antihistamines and corticosteroid. All medication was stopped, with complete resolution in 1 week. Because MAC persisted in cultures, treatment was started with rifampicin and clarithromycin for 1 month, followed by $400 \mathrm{mg}$ of ethambutol. Despite premedication with oral hydroxyzine, she had a similar reaction 3 hours after ethambutol intake. Ethambutol was stopped and she was treated with oral hydroxyzine for two days, with complete resolution in one week.

Despite treatment with rifampicin and clarithromycin, MAC persisted in sputum cultures until November 2010. At this point, the patient was referred to the Drug Allergy Unit. 
TABLE 1: Desensitization protocol for ethambutol.

\begin{tabular}{lcccc}
\hline $\begin{array}{l}\text { Solution } \\
(\mathrm{mg} / \mathrm{mL})\end{array}$ & $\begin{array}{c}\text { Time } \\
(\mathrm{min})\end{array}$ & $\begin{array}{c}\text { Dose } \\
(\mathrm{mL})\end{array}$ & $\begin{array}{c}\text { Dose } \\
(\mathrm{mg})\end{array}$ & $\begin{array}{c}\text { Cumulative } \\
\text { dose }(\mathrm{mg})\end{array}$ \\
\hline \multirow{3}{*}{0.01} & 0 & 1 & 0.01 & 0.01 \\
& 30 & 2 & 0.02 & 0.03 \\
& 60 & 4 & 0.04 & 0.07 \\
& 90 & 8 & 0.08 & 0.13 \\
\hline \multirow{3}{*}{0.1} & 120 & 1 & 0.1 & 0.23 \\
& 150 & 2 & 0.2 & 0.43 \\
& 180 & 4 & 0.4 & 0.83 \\
1 & 210 & 8 & 0.8 & 1.63 \\
\hline \multirow{2}{*}{10} & 240 & 1 & 1 & 2.63 \\
& 270 & 10 & 10 & 12.63 \\
\hline & 300 & 10 & 100 & 112.63 \\
& 330 & 30 & 300 & 412.63
\end{tabular}

Skin tests were performed according to EAACI guidelines [1], using solutions of $1 \mathrm{mg} / \mathrm{mL}$ and $10 \mathrm{mg} / \mathrm{mL}$ [2] prepared from a $400 \mathrm{mg}$ ethambutol tablet. Immediate reading was negative, but a flare reaction with the $1 \mathrm{mg} / \mathrm{mL}$ concentration was present at 6 hours, which resolved at 24 and 72 hours readings. Although skin testing was doubtful, hypersensitivity to ethambutol was diagnosed taking into account the clinical presentation and reproducibility of the reaction upon reexposure.

A two-day tolerance induction protocol was attempted in an outpatient regime, adapted from previous literature reports [3], with a twofold increase every 45 minutes. The first day was completed with $31.6 \mathrm{mg}$ of cumulative dose, with no adverse events. The second day initial dose was $16 \mathrm{mg}$, and at a cumulative dose of $130 \mathrm{mg}$, the patient had a similar reaction to the previously described one. The procedure was modified according to current recommendations [4] to achieve $1200 \mathrm{mg}$ in four days, without success.

As ethambutol was the optimal choice for treatment of MAC, a second protocol was designed based on the twelfth step protocol by Castells et al. [5] (Table 1), with slower increments and premedication with $25 \mathrm{mg}$ of hydroxyzine and $20 \mathrm{mg}$ of oral prednisone. The patient reached $400 \mathrm{mg}$ in the first day and continued on $400 \mathrm{mg}$ during 8 days. Increases to $800 \mathrm{mg}$ and to $1200 \mathrm{mg}$ were done after one week in each dose without adverse reactions. Prednisone and hydroxyzine were discontinued and she was maintained on ethambutol $1200 \mathrm{mg}$ daily for 6 months with good tolerance. In April 2011, the first sputum culture turned negative, but she was unable to complete a full-year treatment due to a confirmed diagnosis of ethambutol-induced optic neuropathy in June 2011. In July 2011 she was diagnosed with hepatic adenocarcinoma of unknown primary and Aspergillus fumigatus was isolated from sputum cultures. Ethambutol was not reintroduced and all antimycobacterial treatment was stopped. The patient is currently followed in Palliative Care, as well as by the Pulmonology Department.

\section{Comments}

Ethambutol is a bacteriostatic antimycobacterial drug that has ocular toxicity due to optic neuritis as the main side effect. Reactions as rash and drug fever have been reported in $0.5 \%$ and $0.3 \%$ of patients, respectively [6], and other hypersensitivity reactions, as ashy dermatosis-like pigmentation [7], lichenoid eruptions [8], pulmonary infiltrates [9], and toxic epidermal necrolysis [10], have been described. Ethambutol is usually used in combined therapy with other antibiotics, which makes diagnosis particularly difficult. The recommended approach in generalized cutaneous reactions is stopping all combined treatment and gradually reintroducing each of the antimycobacterial drugs, to determine the offending drug [11]. Skin and serological tests are usually unreliable, with one paper presenting a positive epicutaneous test [12] and another positive lymphocyte stimulation test [9] to ethambutol.

We present a case of a clinically confirmed hypersensitivity reaction to ethambutol in whom a previously described protocol was ineffective. This new adapted successful desensitization protocol underscores the importance of lower incremental doses with more steps and the use of antihistamines and prednisone to induce immunological tolerization. Because the mechanism of the initial reaction appears to be a hypersensitivity type IV reaction, these reactions have been shown to be responsive to a slower reintroduction of the offending agent, so that reaching the target dose may require few weeks. The mechanism of desensitization may relate to a suboptimal $\mathrm{T}$ cell antigen presentation.

\section{Disclosure}

This case report was briefly presented in a poster session in the 30th Congress of the European Academy of Allergy and Clinical Immunology, 2010, in Allergy 66, Suppl. 94 (2011):381.

\section{Conflict of Interests}

The authors have no funding sources or conflict of interests to declare.

\section{References}

[1] K. Brockow, A. Romano, M. Blanca, J. Ring, W. Pichler, and P. Demoly, "General considerations for skin test procedures in the diagnosis of drug hypersensitivity," Allergy, vol. 57, no. 1, pp. 4551, 2002.

[2] M. P. Lafourcade, M. Martin, X. Revolte et al., "Réaction allergique aux antituberculeux majeurs," Revue Française D'allergologie, vol. 49, no. 6, pp. 496-499, 2009.

[3] S. Rodrigues Carvalho, I. Silva, P. Leiria-Pinto, and J. RosadoPinto, "Rapid oral tolerance induction to isoniazid and pyrazinamide and controlled administration of ethambutol: clinical case," Allergologia et Immunopathologia, vol. 37, no. 6, pp. 336338, 2009. 
[4] J. R. Cernadas, K. Brockow, A. Romano et al., "General considerations on rapid desensitization for drug hypersensitivity-a consensus statement," Allergy, vol. 65, no. 11, pp. 1357-1366, 2010.

[5] M. C. Castells, N. M. Tennant, D. E. Sloane et al., "Hypersensitivity reactions to chemotherapy: outcomes and safety of rapid desensitization in 413 cases," Journal of Allergy and Clinical Immunology, vol. 122, no. 3, pp. 574-580, 2008.

[6] L. L. Brunton, A. Gilman, J. S. Lazo, K. L. Parker, and L. S. Goodman, Goodman \& Gilman's the Pharmacological Basis of therapeutics, McGraw-Hill, 11th edition, 2006.

[7] N. Srivastava, L. Solanki, S. Chand, R. Garbyal, and S. Singh, "Ashy dermatosis-like pigmentation due to ethambutol," Indian Journal of Dermatology, Venereology and Leprology, vol. 74, no. 3, pp. 281-282, 2008.

[8] M. E. Grossman, K. Warren, A. Mady, and K. H. Satin, "Lichenoid eruption associated with ethambutol," Journal of the American Academy of Dermatology, vol. 33, no. 4, pp. 675-676, 1995.

[9] K. Hiraoka, N. Nagata, K. Suzuki et al., "A case of pulmonary reaction with skin eruption showing a positive peripheral lymphocyte stimulation test result for ethambutol," Journal of UOEH, vol. 20, no. 2, pp. 145-151, 1998.

[10] P. S. Pegram Jr., J. D. Mountz, and P. R. O’Bar, "Ethambutolinduced toxic epidermal necrolysis," Archives of Internal Medicine, vol. 141, no. 12, pp. 1677-1678, 1981.

[11] Centers for Disease Control Prevention, "Treatment of tuberculosis, American thoracic society, CDC, and infectious diseases society of America," Morbidity and Mortality Weekly Report, vol. 52, no. 11, article 43, 2003.

[12] R. S. L. A. Bakkum, F. B. D. E. Waard-Van Der Spek, and H. B. Thio, "Delayed-type hypersensitivity reaction to ethambutol and isoniazid," Contact Dermatitis, vol. 46, no. 6, p. 359, 2002. 


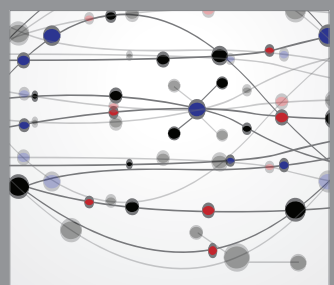

The Scientific World Journal
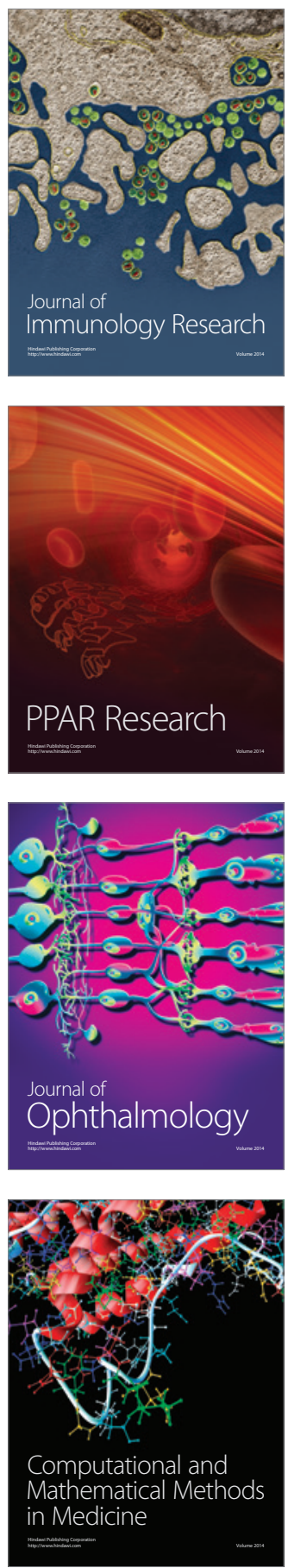

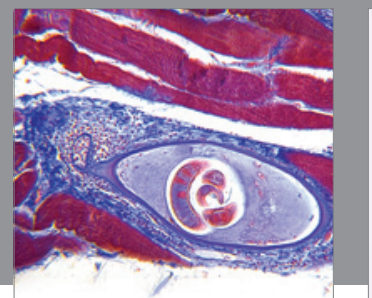

Gastroenterology

Research and Practice
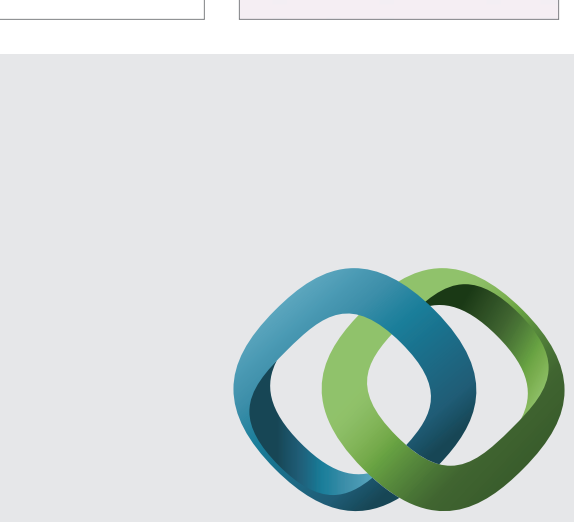

\section{Hindawi}

Submit your manuscripts at

http://www.hindawi.com
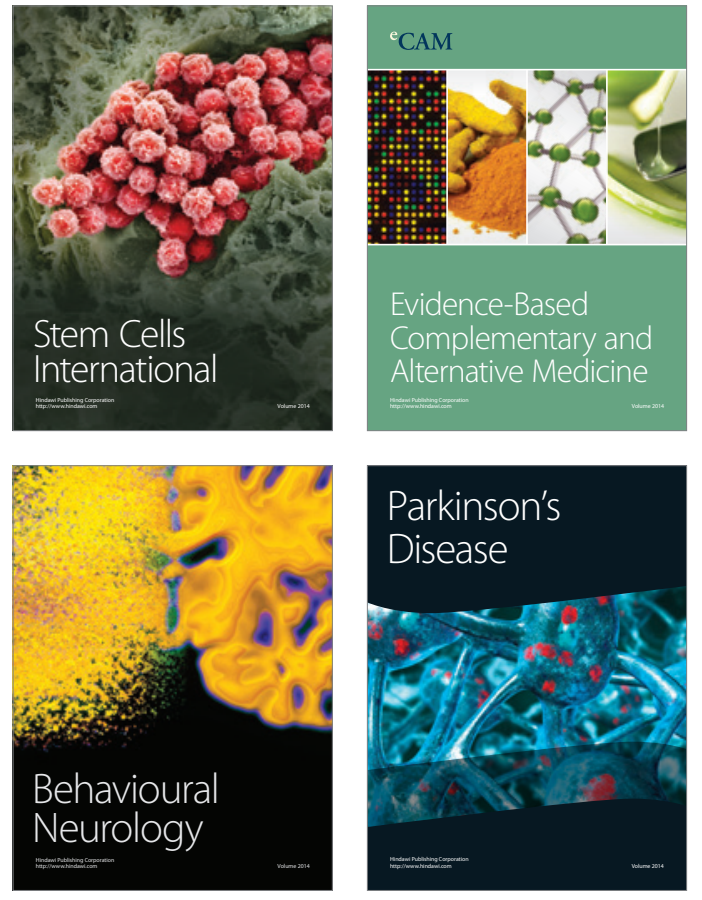
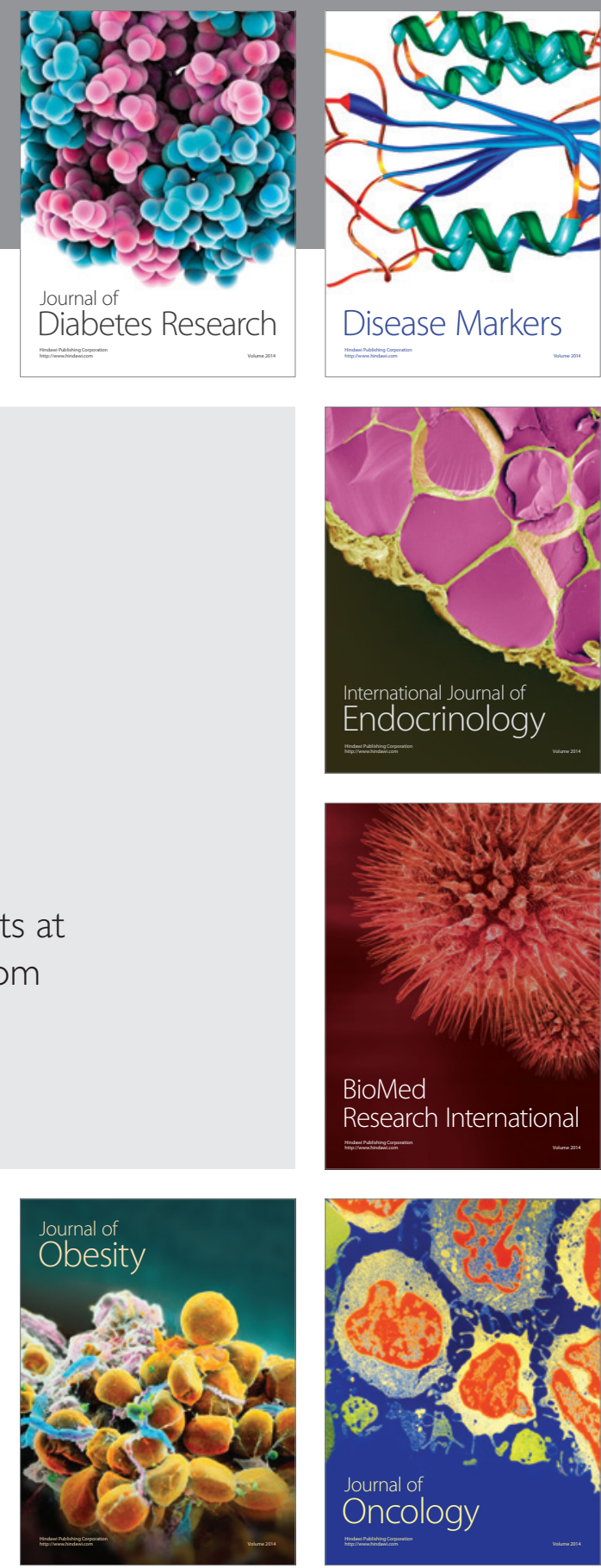

Disease Markers
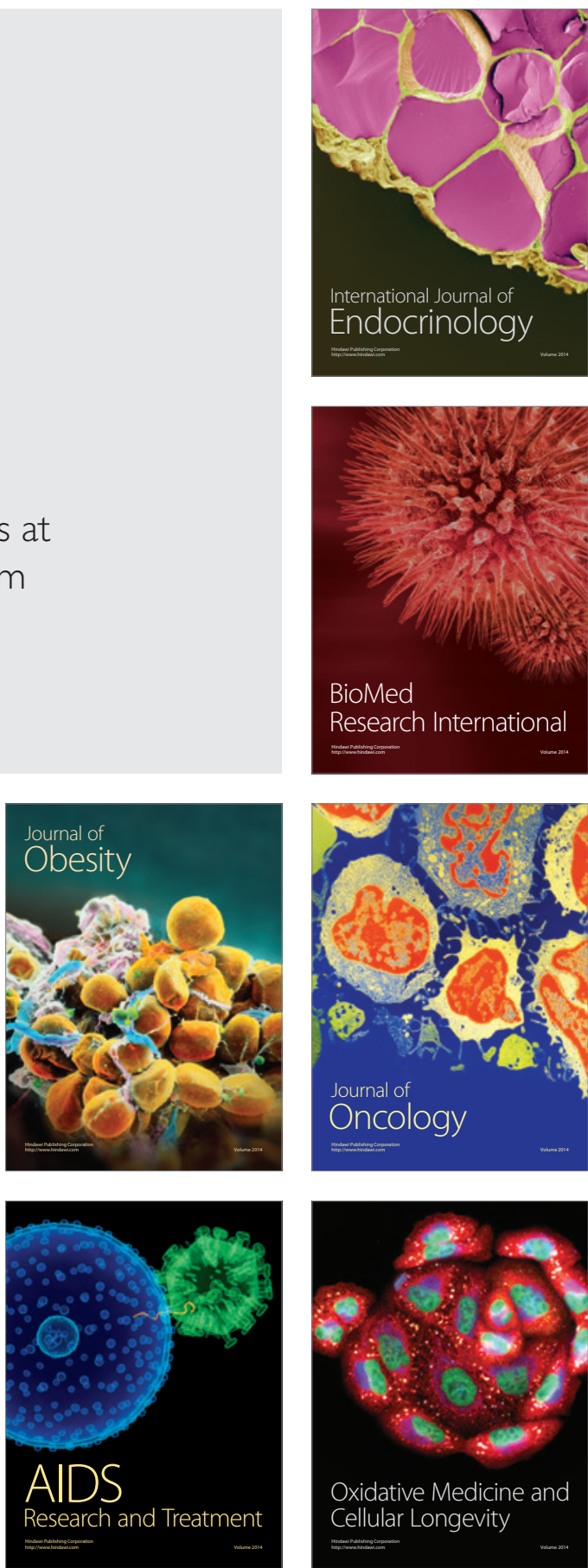